\title{
Impact of Adverse Events on the Progression-Free Survival of Patients with Advanced Hepatocellular Carcinoma Treated with Lenvatinib: A Multicenter Retrospective Study
}

\author{
Takamasa Ohki $^{1}$ (Doki Sato ${ }^{1} \cdot$ Mayuko Kondo ${ }^{1} \cdot$ Eriko Goto $^{2} \cdot$ Takahisa Sato $^{3} \cdot$ Yuji Kondo $^{4} \cdot$ Masatoshi Akamatsu $^{5}$. \\ Shinpei Sato ${ }^{4} \cdot$ Hideo Yoshida $^{6} \cdot$ Yukihiro Koike $^{2} \cdot$ Shuntaro Obi ${ }^{3}$
}

Published online: 11 February 2020

(c) The Author(s) 2020

\begin{abstract}
Background and Objective Experience of the use of lenvatinib (LEN) in the clinical setting remains limited. We conducted this study to elucidate the factors associated with progression-free survival (PFS) in patients with advanced HCC treated with LEN.

Methods In this multicenter retrospective study, we analyzed data on patient characteristics, treatment outcomes, and adverse events (AEs) for 77 patients with advanced hepatocellular carcinoma (HCC). We also analyzed PFS and factors that influence PFS.

Results The response rate to LEN was $29.9 \%$ and the disease control rate was $77.9 \%$. Patients who achieved relative dose intensities of more than $70 \%$ had better outcomes (response rate $45.2 \%$ vs. $11.4 \%, P<0.01$ ). Appetite loss, fatigue, diarrhea, hypertension, and thyroid dysfunction were the most frequent AEs. Twenty-three patients (29.9\%) had grade 3 or 4 AEs. Fifty-two patients (67.5\%) required a dose reduction and 47 (61.0\%) stopped taking the drug due to AEs. The PFS rates at 3,6 , and 12 months were $81.2 \%, 49.8 \%$, and $34.8 \%$, respectively. The median PFS was 5.6 months. Multivariate analysis showed that thyroid dysfunction of grade $\geq 2$ (hazard ratio [HR] 4.57, 95\% confidence interval [CI] 2.05-10.2, $P<0.01$ ), appetite loss (HR 3.58, 95\% CI 1.72-7.52, $P<0.01$ ), and tumor diameter $\geq 40 \mathrm{~mm}$ (HR: 2.27, 95\% CI 1.17-4.40, $P=0.015$ ) were independent factors associated with poor PFS. On the other hand, Child-Pugh class 5A (HR 0.41, 95\% CI 0.19-0.90, $P=0.027$ ) and complete or partial response (HR 0.40, 95\% CI $0.17-0.95, P=0.039$ ) were independent factors associated with better PFS.

Conclusions Thyroid dysfunction and appetite loss after the administration of LEN were independent factors associated with shorter PFS, so these AEs should be carefully managed after administering LEN.
\end{abstract}

Takamasa Ohki

anb72547@nifty.com

1 Department of Gastroenterology, Mitsui Memorial Hospital, 1 Kandaizumicho, Chiyoda-ku, Tokyo 101-8643, Japan

2 Department of Gastroenterology, Kanto Central Hospital, Tokyo, Japan

3 Department of Gastroenterology, Teikyo University Medical Center, Chiba, Japan

4 Department of Gastroenterology and Hepatology, Kyoundo Hospital, Tokyo, Japan

5 Department of Gastroenterology, JR Tokyo General Hospital, Tokyo, Japan

6 Department of Gastroenterology, Japanese Red Cross Medical Center, Tokyo, Japan

\section{Key Points}

Since being introduced recently, lenvatinib (LEN) has been used for unresectable advanced hepatocellular carcinoma (HCC) in the clinical setting.

We found that thyroid dysfunction and appetite loss after the administration of LEN were independent factors associated with shorter progression-free survival.

Relative dose intensity (RDI) was also found to be an important influence on the effect of LEN. 


\section{Introduction}

Hepatocellular carcinoma (HCC) is the second most common cause of cancer-related death worldwide. There were approximately 780,000 deaths from HCC around the world in 2018 [1]. In Japan, a nationwide surveillance program for $\mathrm{HCC}$ has been initiated, leading to the detection of $65 \%$ of all HCC cases at an early stage and facilitating curative surgical or locoregional treatment [2]. Although there is an initial positive response to surgery or locoregional therapy in patients with $\mathrm{HCC}$, the recurrence rates after these treatments are high: $60-80 \%$ over 5 years [3]. Multiple tumor recurrence sometimes occurs during the natural course of the disease; HCC is difficult to cure in such cases and has a poor prognosis. The standard first-line treatment for intermediate $\mathrm{HCC}$ is transcatheter arterial chemoembolization (TACE), which is frequently performed in patients with $\mathrm{HCC}$ who are not eligible for curative treatment and in patients with multiple HCC recurrence [4]. Previous randomized clinical trials have reported that TACE prevents $\mathrm{HCC}$ recurrence for up to 6 months after curative treatment and improves survival rates $[5,6]$. Despite these findings, some patients are unresponsive to TACE, and HCC progression or recurrence may occur rapidly [7-9].

Sorafenib is a multikinase inhibitor that inhibits the Raf-MEK-ERK signaling pathway, which in turn inhibits tumor cell proliferation and blocks vascular endothelial growth factor receptors, preventing neoangiogenesis [10]. Sorafenib, which has been shown to prolong overall survival in HCC patients, has been the only systemic therapy to be used as a first-line treatment for HCC in Asian cohorts for the last 10 years [11]. In the REFLECT trial, lenvatinib (LEN) — an inhibitor of vascular endothelial growth factor receptors $1-3$, fibroblast growth factor receptors 1-4, platelet-derived growth factor receptor $\alpha$, RET (rearranged during transfection), and stem cell factor receptor-was observed to be safe and noninferior to sorafenib in terms of overall survival, progression-free survival (PFS), and time to progression [12].

LEN has been applied in the clinical setting for unresectable advanced HCC as a first-line, second-line, or third-line treatment. As LEN is a novel agent, clinical studies on its effectiveness and prognostic factors remain limited [13-16]. The aim of this study was to evaluate and to determine factors associated with the PFS of patients with advanced HCC treated with LEN.

\section{Methods}

\subsection{Study Design}

This study used a retrospective, multicenter (six hospitals), observational design. A total of 79 consecutive patients with unresectable HCC who were treated with LEN from March 2018 to January 2019 were enrolled. However, only 77 patients were included in the final analysis because 2 patients were excluded for refusing computed tomography or magnetic resonance imaging. Although the inclusion and exclusion criteria for treatment with LEN were based on those used in the REFLECT trial [12], our retrospective study allowed us to include patients previously treated with one or two lines of systemic therapy, patients with a Child-Pugh score of B7, and patients on dialysis. Eligible patients had a measurable target lesion based on the Modified Response Evaluation Criteria in Solid Tumors (mRECIST) [17], a Barcelona Clinic Liver Cancer stage of B or C [18], a Child-Pugh class of A or 7B [19, 20], and an Eastern Cooperative Oncology Group Performance Status score of 0 or 1 [21]. The patients included also had controlled hypertension, sufficient liver function, and adequate bone marrow function. Patients who had a history of sorafenib or regorafenib use or were on maintenance dialysis were also included.

\subsection{Protocol}

Patients received $12 \mathrm{mg} /$ day (body weight $<60 \mathrm{~kg}$ ) or $8 \mathrm{mg}$ / day (for body weight $\geq 60 \mathrm{~kg}$ ) LEN (Eisai Co., Ltd., Tokyo, Japan). Dose interruptions followed by reductions (to $8 \mathrm{mg}$ / day, $4 \mathrm{mg} /$ day, or $4 \mathrm{mg}$ every other day) due to LEN-related adverse events (AEs) were permitted. Eisai Co., Ltd. has prescribed protocols that were introduced in the REFLECT trial [12]. The AEs were graded according to the Common Terminology Criteria for Adverse Events (CTCAE) version 4.0 [22]. Based on the guidelines for administering LEN, the dose was reduced or the treatment was interrupted when a patient experienced an AE of grade 3 or above or if an unacceptable grade $2 \mathrm{AE}$ occurred. The grade for each $\mathrm{AE}$ at its worst during the observation period was recorded. If an AE occurred, the dose was reduced or the treatment was temporarily interrupted until the symptoms improved to grade 1 or 2 , based on the guidelines from Eisai Co., Ltd. The relative dose intensity (RDI) was calculated by dividing the actual dose by the ideal dose for the first 30 days of treatment [23, 24].

\subsection{Follow-Up and Outcomes}

Participants were observed for at least 16 weeks. During safety assessments, vital signs were recorded, hematological and biochemical laboratory testing was performed, and 
urinalysis was conducted at $1,2,4,8$, and 12 weeks after drug administration. AEs were graded according to CTCAE version 4.0 [22]. Therapeutic efficacy was determined at weeks $8-10$ using the mRECIST [17]. The tumor markers $\alpha$-fetoprotein and des-gamma-carboxy prothrombin were measured every 4 weeks. The liver was examined by computed tomography or magnetic resonance imaging using a triple-phase scanning protocol. Baseline data on clinical parameters, AEs, LEN dosages, tumor markers, and radiological findings were collected.

\subsection{Statistical Analyses}

Data are expressed as the median and range (25-75th percentiles). Statistical analyses were performed using the Kaplan-Meier method, the log-rank test, multivariate logistic regression analysis, and multivariate Cox's hazard analysis. A $P$ value of $<0.05$ was considered significant. Factors with a $P$ value of $<0.10$ in the logistic regression and univariate Cox's hazard analysis were included in the multivariate analysis. Data on patient backgrounds, tumor status, RDI $>70 \%$, decreases in tumor marker levels at 30 days, and AEs were utilized in the univariate and multivariate logistic regression analyses. In addition, response (complete/partial response or no response) was added as a parameter in the univariate and multivariate Cox's hazard analyses. Side effects included hand-foot skin reaction, diarrhea, hypertension, appetite loss, fatigue, and thyroid dysfunction. Data processing and analysis were performed using StatView software (version 5.0; SAS Institute, Cary, NC, USA).

\section{Results}

\subsection{Patient Characteristics}

A total of 77 patients with unresectable advanced HCC were included in the analysis. The patients were observed until May 31, 2019. The median dosing period was 173 days. The median patient age was 73.5 years, and 64 patients $(83.1 \%)$ were male. All cases were actual clinical cases of Child-Pugh class 5A, 6A, or 7B. Among the four Child-Pugh 7B patients, one patient had previously been treated with sorafenib and regorafenib, and all four patients had a history of radiofrequency ablation and TACE. Twentythree patients had a history of treatment with tyrosine kinase inhibitors (TKIs). A RDI of $>70 \%$ for the first 30 days of treatment was achieved in 42 patients $(54.5 \%)$. The median maximum tumor size was $36 \mathrm{~mm}$, and 34 patients $(44.2 \%)$ had more than ten tumors. Vascular invasion was observed in 33 patients $(42.9 \%)$, and metastasis occurred in 27 patients $(35.1 \%)$ (Table 1$)$.

\subsection{Treatment Outcomes}

The mRECIST evaluation was successfully performed during weeks 8-10 in all 77 cases. Based on their mRECIST evaluations, two patients achieved a complete response and 21 a partial response (Table 2). Thirty-seven patients achieved stable disease status and the remaining $17 \mathrm{had}$ progressive disease. The response rate was $29.9 \%$ and the disease control rate was $77.9 \%$. There were 42 patients who achieved RDI $>70 \%$ (Table 1), and that cohort of patients had a response rate of $45.2 \%$ (19 patients responded). The remaining 35 patients who did not achieve a RDI of $>70 \%$ had a response rate of $11.4 \%$ (four patients responded). There was a significant difference in response between these two groups $(P<0.01)$.

\subsection{Adverse Events}

AEs occurred in 68 of the 77 patients (88.3\%), and the total number of AEs was 182. The median time to AE occurrence after treatment initiation was 13.5 days (interquartile range: 7-23 days). The adverse events observed are listed in

Table 1 Baseline patient characteristics

\begin{tabular}{|c|c|}
\hline Parameter & Value \\
\hline Dosing period (days) ${ }^{\mathrm{a}}$ & $173(63-248)$ \\
\hline $\begin{array}{l}\text { Median time from initial administration of LEN } \\
\text { (years) }^{\mathrm{a}}\end{array}$ & $3.9(1.2-6.2)$ \\
\hline Age (years $)^{\mathrm{a}}$ & $73.5(68.7-79.5)$ \\
\hline TKI experienced, $n(\%)$ & $23(29.9 \%)$ \\
\hline $\mathrm{RDI}>70 \%, n(\%)$ & $42(54.5 \%)$ \\
\hline Male, $n(\%)$ & $64(83.1 \%)$ \\
\hline HCV-Ab positive, $n(\%)$ & $28(36.4 \%)$ \\
\hline HBV-Ag positive, $n(\%)$ & $12(15.6 \%)$ \\
\hline nBnC-non-alcohol, $n(\%)$ & $33(42.95)$ \\
\hline \multicolumn{2}{|l|}{ Liver function } \\
\hline Child-Pugh 5A, $n(\%)$ & $34(44.2 \%)$ \\
\hline Child-Pugh 6A, $n(\%)$ & $39(50.6 \%)$ \\
\hline Child-Pugh 7B, $n(\%)$ & $4(5.2 \%)$ \\
\hline$>10$ tumors, $n(\%)$ & $34(44.2 \%)^{\mathrm{b}}$ \\
\hline Maximum tumor size (mm) & $36(23-67)^{\mathrm{b}}$ \\
\hline Vascular invasion, $n(\%)$ & $33(42.9 \%)^{\mathrm{c}}$ \\
\hline Metastasis, $n(\%)$ & $27(35.1 \%)$ \\
\hline
\end{tabular}

$T K I$ tyrosine kinase inhibitor, $R D I$ relative dose intensity, $H C V-A b$ hepatitis $\mathrm{C}$ virus antibody, $H B V-A g$ hepatitis $\mathrm{B}$ virus antigen, $n B n C$ non-alcohol non-HBV-related, non-HCV-related, non-alcohol-related liver cirrhosis

${ }^{a}$ Values shown are the median (25th-75th percentile); values given for other parameters are the number of patients (percentage of the 77 patients included in the study)

${ }^{\mathrm{b}}$ Seven patients had HCC with $\geq 50 \%$ liver occupation

${ }^{\mathrm{c}}$ Four patients had a tumor thrombosis of the main portal trunk 
Table 2 Treatment outcomes

\begin{tabular}{lc}
\hline Outcome & Value \\
\hline Complete response & $2(2.6 \%)^{\mathrm{a}, \mathrm{b}}$ \\
Partial response & $21(27.3 \%)^{\mathrm{a}, \mathrm{b}}$ \\
Stable disease & $37(48.1 \%)^{\mathrm{b}}$ \\
Progressive disease & $17(22.1 \%)$ \\
\hline
\end{tabular}

Values shown are the number of patients (percentage of the 77 patients included in the study)

${ }^{a}$ Based on these values, the response rate was $29.9 \%$

${ }^{\mathrm{b}}$ Based on these values, the disease control rate was $77.9 \%$

Table 3 Adverse events (AEs)

\begin{tabular}{lccll}
\hline $\mathrm{AE}$ & $\begin{array}{c}\text { Grade 1 } \\
(n=89)\end{array}$ & $\begin{array}{c}\text { Grade 2 } \\
(n=70)\end{array}$ & $\begin{array}{l}\text { Grade 3 } \\
(n=21)\end{array}$ & $\begin{array}{l}\text { Grade 4 } \\
(n=2)\end{array}$ \\
\hline Hand-foot skin reaction & 16 & 5 & 1 & 0 \\
Itching and rash & 4 & 2 & 0 & 0 \\
Diarrhea & 16 & 7 & 0 & 0 \\
Hypertension & 12 & 11 & 0 & 0 \\
Liver dysfunction & 0 & 1 & 2 & 1 \\
Appetite loss & 15 & 15 & 1 & 0 \\
Fatigue & 12 & 5 & 9 & 0 \\
Thyroid dysfunction & 6 & 14 & 0 & 0 \\
Proteinuria & 2 & 2 & 4 & 0 \\
Hepatic encephalopathy & 0 & 2 & 1 & 0 \\
Others & 6 & 6 & 3 & 1
\end{tabular}

Values shown are the number of adverse events of a particular type and grade. $n$ is the total number of AEs of a particular grade

Table 3. Appetite loss was the most frequent AE (31 occurrences), followed by fatigue ( 26 occurrences), hypertension (23 occurrences), and diarrhea (23 occurrences). Almost all of the other AEs were of grade 1 or 2 . The number of grade 1 AEs was 89 and the number of grade 2 AEs was 70 . Twenty-three patients (29.9\%) had grade 3 or 4 AEs. Two grade 4 AEs were observed: a case of liver dysfunction and a case of chronic heart failure. Ultimately, 52 patients (67.5\%) required a dose reduction and 47 patients $(61.0 \%)$ discontinued their treatment due to AEs. The most common reason for dose reduction or discontinuation was appetite loss. Cessation or dose reduction of LEN occurred in 28 of the 31 patients $(90.3 \%)$ in our appetite loss ( $\geq$ grade 1$)$ group and in 34 of the 46 patients (73.9\%) in our no appetite loss group $(P=0.07)$. Cessation or dose reduction of LEN occurred in 12 of the 14 patients $(85.7 \%)$ in our thyroid dysfunction ( $\geq$ grade 2 ) group and in 50 of the 63 patients $(79.4 \%$ ) in our thyroid dysfunction (<grade 2$)$ group $(P=0.59)$. The initial dose was reduced in 12 patients based on the judgment of the primary doctor. Excluding these 12 patients, we compared the frequency of AEs between the Child-Pugh 5A patients $(n=31)$ and the Child-Pugh 6A patients $(n=32)$. There was no significant difference in the rate of occurrence of grade $\geq 2$ AEs between these two groups (24 patients [77.4\%] vs. 24 patients [75.0\%], $P=0.83$ ).

\subsection{Factors Contributing to Treatment Outcomes}

The multivariate analysis revealed that Child-Pugh 5A (odds ratio [OR] 4.77, 95\% confidence interval [CI] 1.31-17.4, $P=0.018$ ), RDI $>70 \%$ (OR 5.61, 95\% CI 1.40-22.5, $P=0.015)$, and decrease in AFP level (OR 10.3, 95\% CI 1.81-58.7, $P<0.01)$ were independent factors associated with the response to LEN (Table 4).

\subsection{Progression-Free Survival}

The estimated median PFS was 5.6 months (Fig. 1). The PFS rates at 3,6 , and 12 months were $81.2 \%, 49.8 \%$, and $34.8 \%$, respectively. Patients with a thyroid dysfunction grade of $\geq 2$ presented significantly worse PFS (median PFS: 8.0 vs. 4.2 months, $P=0.014$ ) (Fig. 2). Patients who experienced a loss of appetite tended to have a worse PFS (median PFS: 8.7 vs. 5.2 months, $P=0.075$ ) (Fig. 3). Patients with a tumor size of $\geq 40 \mathrm{~mm}$ exhibited significantly worse PFS (median PFS: 8.3 vs. 4.4 months, $P=0.033$ ) (Fig. 4). On the other hand, patients who had good liver function (Child-Pugh class 5A) presented significantly better PFS (median PFS: 9.4 vs. 4.7 months, $P<0.01$ ) (Fig. 5). We also performed a subanalysis comparing the PFS of Child-Pugh class 5A patients with the PFS of Child-Pugh class 6A patients. There was a significant difference in PFS between the two groups (median PFS: 9.4 vs. 4.8 months, $P=0.023$ ). Patients with a RDI of $>70 \%$ exhibited significantly better PFS (median PFS: 9.3 vs. 4.7 months, $P=0.016$ ) (Fig. 6). Patients who showed a complete or partial response also presented significantly better PFS (median PFS: 9.3 vs. 4.7 months, $P=0.046$ ) (Fig. 7). We did not assess the overall survival because only 12 patients $(15.6 \%)$ died during the follow-up period.

\subsection{Factors Contributing to Progression-Free Survival}

The multivariate analysis revealed that a thyroid dysfunction grade of $\geq 2$ (hazard ratio [HR] 4.57, 95\% CI 2.05-10.2, $P<0.01$ ), appetite loss (HR 3.58, 95\% CI 1.72-7.52, $P<0.01$ ), and a tumor diameter of $\geq 40 \mathrm{~mm}$ (HR $2.27,95 \%$ CI 1.17-4.40, $P=0.015)$ were independent factors associated with poor PFS. In contrast, Child-Pugh class 5A (HR $0.41,95 \%$ CI $0.19-0.90, P=0.027)$ and complete or partial response (HR 0.40, 95\% CI $0.17-0.95, P=0.039$ ) were independent factors associated with a better PFS (Table 5). 
Table 4 Univariate and multivariate logistic regression analysis of factors associated with the response to LEN

\begin{tabular}{llrrl}
\hline Variables & OR $(95 \%$ CI $):$ unadjusted & \multicolumn{1}{c}{$p$} & OR $(95 \%$ CI): adjusted & $p$ \\
\hline Male vs. female & $0.95(0.26-3.46)$ & 0.94 & & \\
Age (/year) & $1.00(0.94-1.05)$ & 0.86 & & \\
HCV-Ab positive & $0.37(0.12-1.16)$ & 0.09 & $0.73(0.18-3.00)$ & 0.66 \\
Child-Pugh 5A & $6.17(2.07-18.4)$ & $<0.01$ & $4.77(1.31-17.4)$ & 0.018 \\
TKI experienced & $0.56(0.18-1.74)$ & 0.31 & & \\
RDI $>$ 70\% & $6.40(1.92-21.4)$ & $<0.01$ & $5.61(1.40-22.5)$ & \\
HFS $\geq$ grade 2 & $1.19(0.20-7.00)$ & 0.85 & \\
Diarrhea $\geq$ grade 2 & $0.76(0.14-4.09)$ & 0.75 & & \\
Hypertension $\geq$ grade 2 & $0.75(0.18-3.07)$ & 0.69 & & \\
Thyroid dysfunction $\geq$ grade 2 & $2.02(0.61-6.71)$ & 0.25 & & \\
Fatigue grade $\geq$ grade 2 & $1.09(0.33-3.57)$ & 0.89 & & \\
Appetite loss grade $\geq$ grade 2 & $1.71(0.57-5.18)$ & 0.34 & & \\
Metastasis & $1.07(0.38-2.98)$ & 0.90 & & \\
Tumor size $\geq 40$ mm & $0.89(0.33-2.38)$ & 0.82 & & $<0.01$ \\
Number of tumors $>10$ & $0.96(0.36-2.57)$ & 0.94 & & \\
Vascular invasion & $1.71(0.64-4.59)$ & 0.28 & & \\
Decrease in AFP level & $11.3(2.41-53.1)$ & $<0.01$ & $10.3(1.81-58.7)$ & \\
Decrease in DCP level & $3.01(1.04-8.67)$ & 0.041 & $1.77(0.45-6.96)$ & \\
\hline
\end{tabular}

$H C V$ - $A b$ hepatitis $\mathrm{C}$ virus antibody, TKI tyrosine kinase inhibitor, $R D I$ relative dose intensity, $H F S$ handfoot skin reaction, $A F P \alpha$-fetoprotein, $D C P$ des-gamma-carboxy prothrombin, $O R$ odds ratio, $C I$ confidence interval

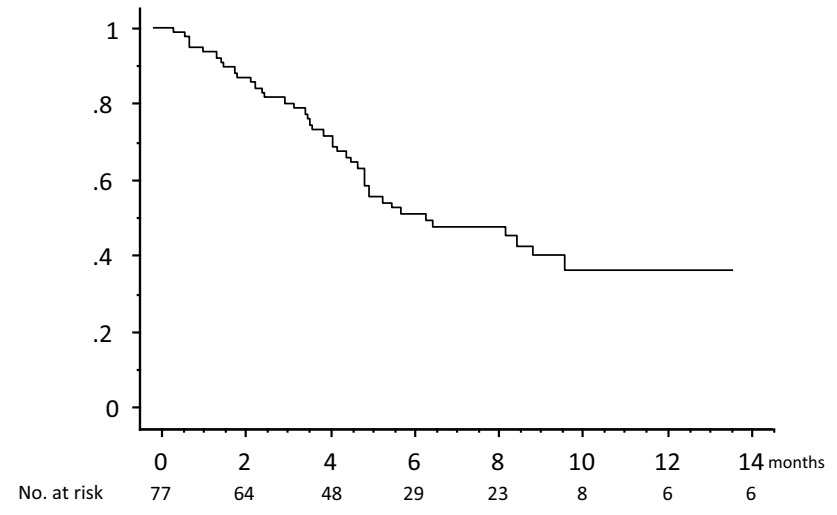

Fig. 1 PFS of all patients following LEN treatment initiation. The estimated median PFS was 5.6 months. PFS progression-free survival

No other factors had a significant effect in the multivariate analysis.

\section{Discussion}

LEN is associated with a higher response rate than other TKIs (sorafenib and regorafenib) [11, 12, 25]. In previous studies, the response rate to LEN (based on the mRECIST) reached $40-60 \%[12,14]$. In our study, the response rate was $29.9 \%$, which-although higher than the response rates to previous TKIs-was lower than those reported previously

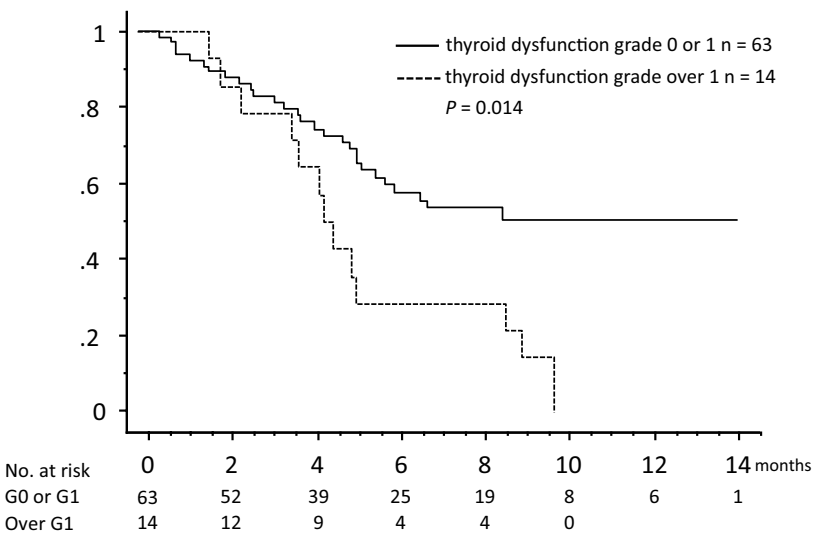

Fig. 2 PFS of patients with a thyroid dysfunction grade of $\geq 2$ and the PFS of patients with a thyroid dysfunction grade of $<2$. Patients with a thyroid dysfunction grade of $\geq 2$ presented a significantly worse median PFS ( 8.0 vs. 4.2 months, $P=0.014$ by log-rank test). $P F S$ progression-free survival

for LEN $[12,14]$. This result could be due to differences in patient characteristics. Only $44.2 \%$ of all patients were of Child-Pugh class 5A, and around half of those patients had advanced HCC (33 patients had vascular invasion and 27 patients had metastasis); moreover, 23 patients (29.9\%) were treated with LEN as third-line chemotherapy.

Although the occurrence rate and distribution of AEs in this study were similar to those reported previously [12, 


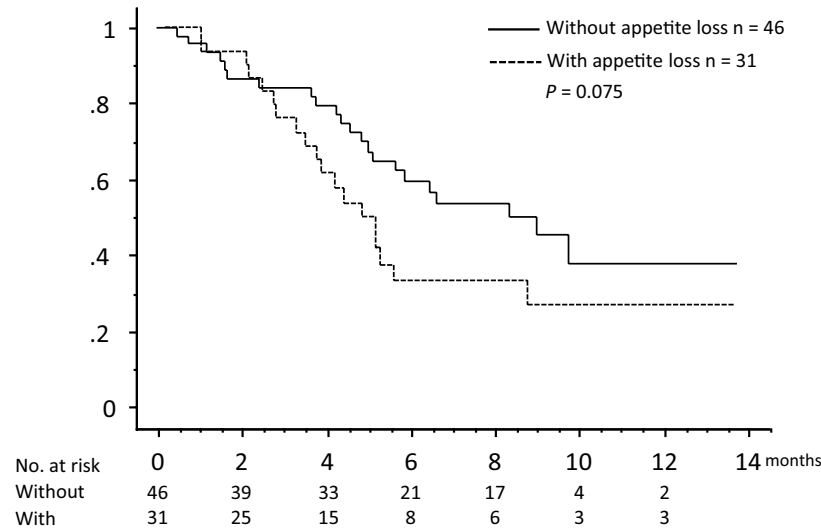

Fig. 3 PFS of patients with appetite loss (of any grade) and the PFS of patients with no appetite loss. Patients with appetite loss tended to have a worse median PFS (5.2 vs. 8.7 months, $P=0.075$ by log-rank test). $P F S$ progression-free survival

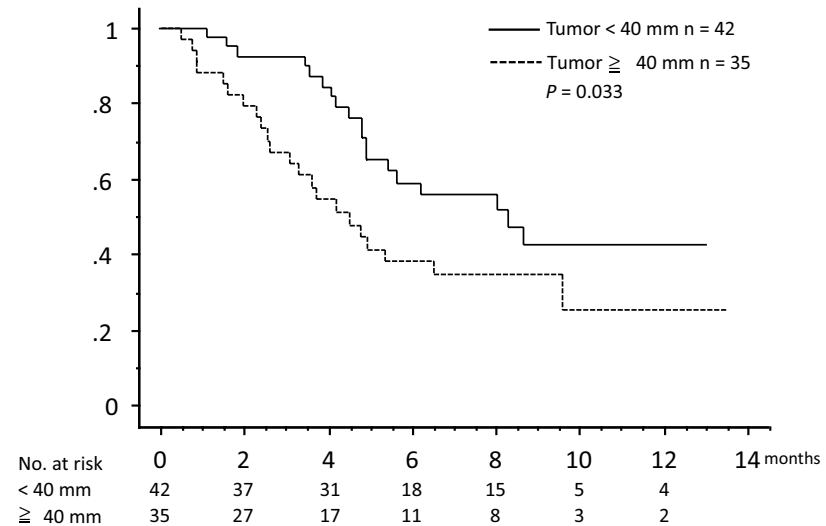

Fig. 4 PFS of patients with a tumor size of $\geq 40 \mathrm{~mm}$ and the PFS of patients with a tumor size of $<40 \mathrm{~mm}$. Patients with a tumor size of $\geq 40 \mathrm{~mm}$ exhibited a significantly worse median PFS (8.3 vs. 4.4 months, $P=0.033$ by log-rank test). $P F S$ progression-free survival

15], the most frequent $\mathrm{AE}$ was appetite loss (31 patients [40.3\%]), followed by fatigue (26 patients [33.8\%]). These AEs proved difficult to control compared to hand-foot skin reactions, diarrhea, hypertension, and thyroid dysfunction. Only a limited number of agents that target these symptoms are available: dexamethasone, domperidone, metoclopramide, and some Chinese herbal medicines (Juzentaihoto, Hochuekkito, and Rikkunshito). In total, 52 of the 77 patients $(67.5 \%)$ required a LEN dose reduction, and 47 of the 77 patients (61.0\%) ultimately stopped taking LEN altogether; both of these rates were higher than those for other TKIs (sorafenib and regorafenib). Thus, AEs associated with LEN should be carefully monitored and treated to promote LEN treatment continuation.

The overall response rate was $29.9 \%$, which was lower than that in the REFLECT trial. This may be due to

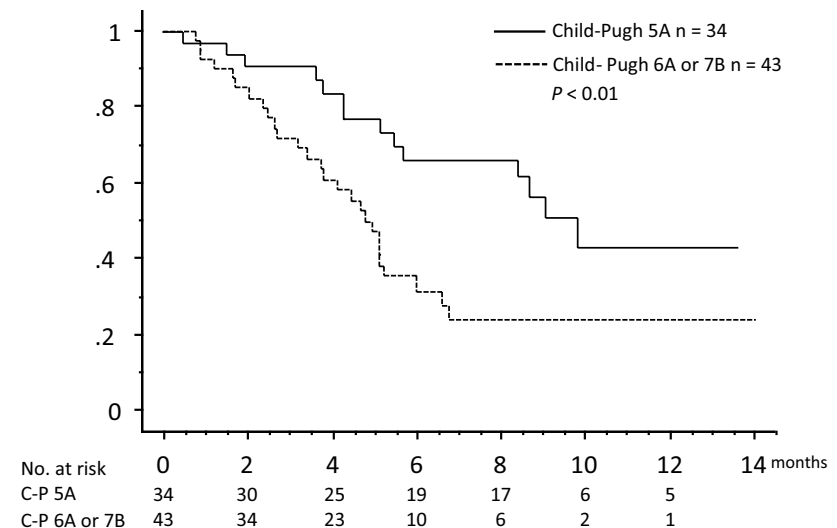

Fig. 5 PFS of patients with a Child-Pugh class of 5A and the PFS of patients with a Child-Pugh class of $6 \mathrm{~A}$ or $7 \mathrm{~B}$. Child-Pugh class $5 \mathrm{~A}$ patients exhibited a better median PFS (9.4 vs. 4.7 months, $P<0.01$ by log-rank test). $P F S$ progression-free survival

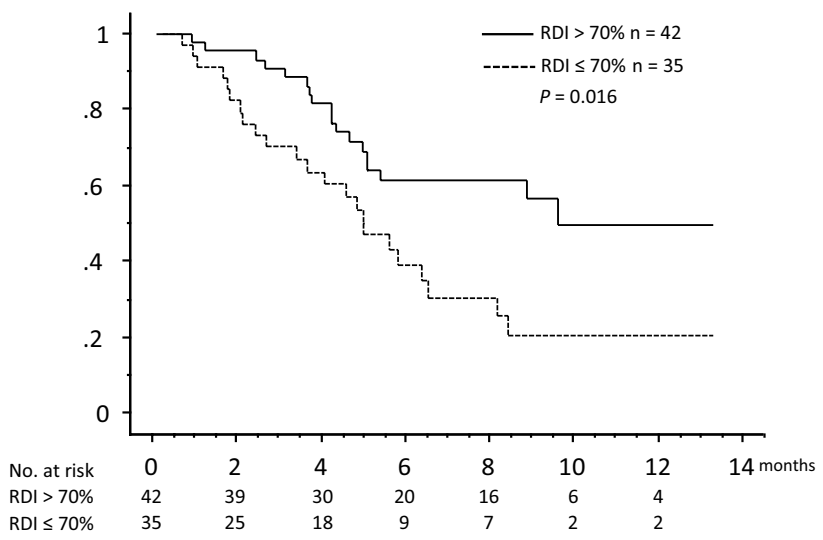

Fig. 6 PFS of patients with RDI $>70 \%$ and the PFS of patients with $\mathrm{RDI} \leq 70 \%$. Patients with RDI $>70 \%$ presented a significantly higher PFS (median PFS: 9.3 vs. 4.8 months, $P=0.016$ by log-rank test). $P F S$ progression-free survival, $R D I$ relative dose intensity

differences in patient background between the studies, as our study included Child-Pugh 7B patients, patients with tumor thrombosis of the main portal trunk, a tumor burden of over $50 \%$ of the whole liver, and patients on dialysis. Logistic regression analysis revealed factors that were associated with the response to LEN. RDI was found to be significantly related to complete or partial response. Recently, the therapeutic effects of LEN at particular dosages have been reported [23, 24]. Those works concluded that achieving a sufficient RDI was crucial to therapeutic efficacy. Our data also showed that those who achieved a RDI of $>70 \%$ during the first 30 days of treatment had a significantly higher response rate than those who did not achieve that RDI level (45.2\% vs. $11.4 \%, P<0.01)$, in agreement with the previously mentioned reports. 


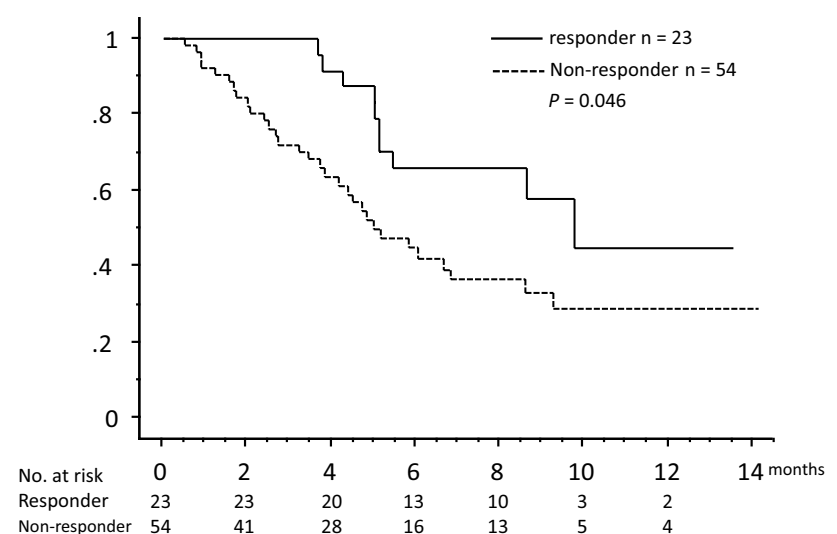

Fig. 7 PFS of patients who showed a complete or partial response and the PFS of patients who did not show a complete or partial response. Patients who showed a complete or partial response presented a significantly higher PFS (median PFS: 9.3 vs. 4.7 months, $P=0.046$ by log-rank test). $P F S$ progression-free survival
The median PFS in our study was 5.6 months, which was shorter than that found in the REFLECT study [12]. This result may also be due to differences in patient characteristics between the studies. Kaplan-Meier curves of PFS stratified by AEs, tumor diameter, and liver function revealed factors associated with PFS that were subsequently confirmed to be independent predictive factors in the multivariate analysis. Based on the proportion of Child-Pugh class 5A cases and the tumor diameters (which represent the tumor burden), our results appear to be quite reasonable [16]. However, the mechanisms underlying the associations of appetite loss (any grade) and thyroid dysfunction ( $\geq$ grade 2 ) with PFS are unclear. Appetite loss could lead to a deterioration in nutritional status; the serum albumin level after administering LEN for 1 month was $3.2 \mathrm{~g} / \mathrm{dl}$ in the appetite loss group and $3.5 \mathrm{~g} / \mathrm{dl}$ in the no appetite loss group $(P=0.013)$. The albumin level was associated with Child-Pugh class, and a decrease in Child-Pugh class reduced the likelihood of continuing with LEN treatment. Also, the treatment period tended to be shorter in the appetite loss group (147 days vs. 183 days in the no appetite loss group, $P=0.16$ ). Thyroid
Table 5 Univariate and multivariate Cox's hazard analysis of factors associated with PFS

\begin{tabular}{lllll}
\hline Variables & HR $(95 \%$ CI): unadjusted & $p$ & HR $(95 \%$ CI): adjusted & $p$ \\
\hline Male vs. female & $1.16(0.54-2.52)$ & 0.70 & & \\
Age (/year) & $1.00(0.96-1.03)$ & 0.88 & & \\
HCV-Ab positive & $1.22(0.65-2.30)$ & 0.53 & & 0.027 \\
Child-Pugh 5A & $0.42(0.22-0.82)$ & 0.01 & $0.41(0.19-0.90)$ & \\
TKI experienced & $0.92(0.48-1.79)$ & 0.82 & & 0.21 \\
RDI $>$ 70\% & $0.48(0.26-0.88)$ & 0.019 & $0.66(0.34-1.27)$ & \\
HFS (any grade) & $0.61(0.30-1.24)$ & 0.17 & & \\
HFS $\geq$ grade 2 & $0.37(0.09-1.54)$ & 0.17 & & \\
Diarrhea (any grade) & $0.68(0.33-1.39)$ & 0.29 & & \\
Diarrhea $\geq$ grade 2 & $0.31(0.07-1.28)$ & 0.11 & & \\
Hypertension (any grade) & $0.95(0.49-1.81)$ & 0.86 & & \\
Hypertension $\geq$ grade 2 & $0.47(0.18-1.20)$ & 0.12 & & \\
Thyroid dysfunction (any grade) & $1.68(0.89-3.17)$ & 0.11 & & \\
Thyroid dysfunction $\geq$ grade 2 & $2.24(1.16-4.35)$ & 0.017 & $4.57(2.05-10.2)$ & \\
Fatigue (any grade) & $1.28(0.68-2.39)$ & 0.44 & & \\
Fatigue $\geq$ grade 2 & $0.96(0.46-2.02)$ & 0.92 & & \\
Appetite loss (any grade) & $1.74(0.94-3.23)$ & 0.079 & $3.58(1.2-7.52)$ & \\
Appetite loss $\geq$ grade 2 & $1.73(0.88-3.40)$ & 0.11 & & \\
Metastasis & $1.17(0.62-2.20)$ & 0.64 & \\
Tumor size $\geq 40$ mm & $1.93(1.04-3.57)$ & 0.037 & $2.27(1.17-4.40)$ & \\
Number of tumors $>10$ & $1.18(0.63-2.18)$ & 0.61 & & \\
Vascular invasion & $1.04(0.56-1.92)$ & 0.91 & & \\
Complete or partial response & $0.49(0.24-1.01)$ & 0.051 & $0.40(0.17-0.95)$ & \\
Decrease in AFP level & $1.13(0.59-2.19)$ & 0.71 & & \\
Decrease in DCP level & $0.82(0.40-1.69)$ & 0.60 & & \\
\hline & & & \\
\end{tabular}

$H C V$ - $A b$ hepatitis $\mathrm{C}$ virus antibody, TKI tyrosine kinase inhibitor, $R D I$ relative dose intensity, $H F S$ handfoot skin reaction, $A F P \alpha$-fetoprotein, $D C P$ des-gamma-carboxy prothrombin, $H R$ hazard ratio, $C I$ confidence interval 
dysfunction has been reported to be an AE that is positively related to PFS [13]. However, in the current study, thyroid dysfunction ( $\geq$ grade 2 ) was negatively related to PFS. Discontinuation or dose reduction of LEN was observed in 12 of the 14 patients $(85.7 \%)$ in our thyroid dysfunction ( $\geq$ grade 2) group and in 50 of the 63 patients (79.4\%) in our thyroid dysfunction ( $<$ grade 2$)$ group $(P=0.59)$. It is unclear why thyroid dysfunction was related to poor PFS, so this requires further study [13]. Although RDI $>70 \%$ was found to be an independent factor linked to complete or partial response, it did not retain statistical significance in the multivariate analysis of factors associated with PFS. However, complete or partial response was a significant influence on PFS, and RDI $>70 \%$ was observed to be one of the factors associated with responders. Thus, it appears that a RDI of $>70 \%$ is linked to response to LEN.

The limitations of this study include its retrospective nature and the small number of participants. It also utilized a multicenter design, and the mRECIST criteria are sometimes difficult to use in clinical practice and require a high level of expertise, implying that the quality and reliability of evaluation may not have been consistently high. Also, as LEN is a novel agent, the observation period was necessarily short, and we could not determine the overall survival rate.

This clinical study has highlighted the influence of appetite loss and thyroid dysfunction on PFS. In particular, appetite loss was difficult to control and was closely related to reduced serum albumin levels. This AE must be managed to ensure that an appropriate dose of LEN is maintained over a sufficient period to achieve favorable outcomes.

\section{Conclusions}

Our study revealed that thyroid dysfunction and appetite loss after the administration of LEN are independent factors that shorten progression-free survival. These AEs must be managed in order to maintain the relative dose intensity and therefore achieve better outcomes.

Acknowledgements I sincerely appreciate all of the coauthors of this study, whose enormous support and insightful comments were invaluable.

Author Contributions All of the authors collected data. The first author analyzed the data and wrote this article.

\section{Compliance with Ethical Standards}

Funding This study received no funding.

Conflict of interest Takamasa Ohki has received speaking fees from Bayer Yakuhin and Eisai Pharmaceutical Co., Ltd. The other authors have nothing to disclose.
Ethical Approval Formal consent was not required for this type of study. The protocol was approved by the Institutional Ethics Committee of Mitsui Memorial Hospital. A retrospective analysis of records stored in a database was performed, and approval was obtained from the Ministry of Health and Welfare, Japan. All procedures complied with the Declaration of Helsinki.

Informed Consent Written informed consent was obtained from all patients when they began treatment, but was not required for the analyses presented herein because this was a retrospective study.

Open Access This article is licensed under a Creative Commons Attribution-NonCommercial 4.0 International License, which permits any non-commercial use, sharing, adaptation, distribution and reproduction in any medium or format, as long as you give appropriate credit to the original author(s) and the source, provide a link to the Creative Commons licence, and indicate if changes were made. The images or other third party material in this article are included in the article's Creative Commons licence, unless indicated otherwise in a credit line to the material. If material is not included in the article's Creative Commons licence and your intended use is not permitted by statutory regulation or exceeds the permitted use, you will need to obtain permission directly from the copyright holder.To view a copy of this licence, visit $\mathrm{http} / / /$ creativecommons.org/licenses/by-nc/4.0/.

\section{References}

1. Bray F, Ferlay J, Soerjomataram I, Siegel RL, Torre LA, Jemal A. Global cancer statistics 2018: GLOBOCAN estimates of incidence and mortality worldwide for 36 cancers in 185 countries. CA Cancer J Clin. 2018;68:394-424.

2. Ikai I, Arii S, Okazaki M, Okita K, Omata M, Kojiro M, Takayasu $\mathrm{K}$, et al. Report of the 17th Nationwide Follow-up Survey of Primary Liver Cancer in Japan. Hepatol Res. 2007;37:676-91.

3. Hasegawa K, Makuuchi M, Takayama T, Kokudo N, Arii S, Okazaki M, Okita K, et al. Surgical resection vs. percutaneous ablation for hepatocellular carcinoma: a preliminary report of the Japanese nationwide survey. J Hepatol. 2008;49:589-94.

4. Takayasu K, Arii S, Ikai I, Omata M, Okita K, Ichida T, Matsuyama Y, et al. Prospective cohort study of transarterial chemoembolization for unresectable hepatocellular carcinoma in 8510 patients. Gastroenterology. 2006;131:461-9.

5. Llovet JM, Real MI, Montana X, Planas R, Coll S, Aponte J, Ayuso C, et al. Arterial embolisation or chemoembolisation versus symptomatic treatment in patients with unresectable hepatocellular carcinoma: a randomised controlled trial. Lancet. 2002;359:1734-9.

6. Cheng HY, Wang X, Chen D, Xu AM, Jia YC. The value and limitation of transcatheter arterial chemoembolization in preventing recurrence of resected hepatocellular carcinoma. World J Gastroenterol. 2005; 11:3644-6.

7. Kokudo N, Hasegawa K, Akahane M, Igaki H, Izumi N, Ichida T, Uemoto $\mathrm{S}$, et al. Evidence-based clinical practice guidelines for hepatocellular carcinoma: The Japan Society of Hepatology 2013 update (3rd JSH-HCC Guidelines). Hepatol Res. 2015;45:1109.

8. Ohki T, Kondo M, Karasawa Y, Kawamura S, Maeshima S, Kojima K, Seki M, et al. Evaluation of the efficacy of sorafenib on overall survival in patients with hepatocellular carcinoma using FT rate: a devised index. Adv Ther. 2017;34:1097-108.

9. Ohki T, Sato K, Yamagami M, Ito D, Yamada T, Kawanishi K, Kojima K, et al. Efficacy of transcatheter arterial chemoembolization followed by sorafenib for intermediate/advanced 
hepatocellular carcinoma in patients in Japan: a retrospective analysis. Clin Drug Investig. 2015;35:751-9.

10. Wilhelm SM, Carter C, Tang L, Wilkie D, McNabola A, Rong H, Chen C, et al. BAY 43-9006 exhibits broad spectrum oral antitumor activity and targets the RAF/MEK/ERK pathway and receptor tyrosine kinases involved in tumor progression and angiogenesis. Cancer Res. 2004;64:7099-109.

11. Llovet JM, Ricci S, Mazzaferro V, Hilgard P, Gane E, Blanc JF, de Oliveira AC, et al. Sorafenib in advanced hepatocellular carcinoma. N Engl J Med. 2008;359:378-90.

12. Kudo M, Finn RS, Qin S, Han KH, Ikeda K, Piscaglia F, Baron A, et al. Lenvatinib versus sorafenib in first-line treatment of patients with unresectable hepatocellular carcinoma: a randomised phase 3 non-inferiority trial. Lancet. 2018;391:1163-73.

13. Koizumi Y, Hirooka M, Hiraoka A, Ochi H, Tanaka T, Yukimoto A, Imai Y, et al. Lenvatinib-induced thyroid abnormalities in unresectable hepatocellular carcinoma. Endocr J. 2019;66:787-92.

14. Hiraoka A, Kumada T, Kariyama K, Takaguchi K, Itobayashi E, Shimada N, Tajiri K, et al. Therapeutic potential of lenvatinib for unresectable hepatocellular carcinoma in clinical practice: multicenter analysis. Hepatol Res. 2019;49:111-7.

15. Hiraoka A, Kumada T, Kariyama K, Takaguchi K, Atsukawa M, Itobayashi E, Tsuji K, et al. Clinical features of lenvatinib for unresectable hepatocellular carcinoma in real-world conditions: multicenter analysis. Cancer Med. 2019;8:137-46.

16. Hiraoka A, Kumada T, Atsukawa M, Hirooka M, Tsuji K, Ishikawa T, Takaguchi K, et al. Prognostic factor of lenvatinib for unresectable hepatocellular carcinoma in real-world conditionsmulticenter analysis. Cancer Med. 2019;8:3719.
17. Lencioni R, Llovet JM. Modified RECIST (mRECIST) assessment for hepatocellular carcinoma. Semin Liver Dis. 2010;30:52-60.

18. Llovet JM, Bru C, Bruix J. Prognosis of hepatocellular carcinoma: the BCLC staging classification. Semin Liver Dis. 1999;19:329-38.

19. Child CG, Turcotte JG. Surgery and portal hypertension. Major Probl Clin Surg. 1964;1:1-85.

20. Pugh RN, Murray-Lyon IM, Dawson JL, Pietroni MC, Williams R. Transection of the oesophagus for bleeding oesophageal varices. Br J Surg. 1973;60:646-9.

21. Oken MM, Creech RH, Tormey DC, Horton J, Davis TE, McFadden ET, Carbone PP. Toxicity and response criteria of the Eastern Cooperative Oncology Group. Am J Clin Oncol. 1982;5:649-55.

22. National Institutes of Health. Common Terminology Criteria for Adverse Events v.4.0 (CTCAE). Bethesda: NIH; 2009.

23. Sasaki R, Fukushima M, Haraguchi M, Miuma S, Miyaaki H, Hidaka M, Eguchi S, et al. Response to lenvatinib is associated with optimal relative dose intensity in hepatocellular carcinoma: experience in clinical settings. Cancers (Basel). 2019;11:1769.

24. Takahashi A, Moriguchi M, Seko Y, Ishikawa H, Yo T, Kimura $\mathrm{H}$, Fujii H, et al. Impact of relative dose intensity of early-phase lenvatinib treatment on therapeutic response in hepatocellular carcinoma. Anticancer Res. 2019;39:5149-56.

25. Bruix J, Qin S, Merle P, Granito A, Huang YH, Bodoky G, Pracht $\mathrm{M}$, et al. Regorafenib for patients with hepatocellular carcinoma who progressed on sorafenib treatment (RESORCE): a randomised, double-blind, placebo-controlled, phase 3 trial. Lancet. 2017:389:56-66. 